\title{
HOT WIRE METHOD FOR THE THERMAL CHARACTERIZATION OF MATERIALS: INVERSE PROBLEM APPLICATION
}

\author{
S. André \\ LEMTA-UMR CNRS 7563 \\ 2 av de la forêt de Haye \\ BP 160,54504 \\ An experimental set-up of the hot wire method is presented. The present design \\ allows the measurement of the temperatures at two different points on the heating \\ wire with an acquisition system that performs measurements at a frequency of $1 \mathrm{kHz}$ \\ with a 12 bit numerical converter. An analytical solution for the direct model for the \\ time dependent problem of heat transfer is employed. It is based on the quadrupole \\ method which basically consists in a transfer matrix approach which is possible \\ through the use of Laplace transforms. It extends the electrical analogy of heat \\ transfer problems using the notion of impedance, and allows to take into account the \\ thermal behavior of the wire, as well as contact resistance and heat loss effects in a \\ very simple straightforward way. In the identification process carried on the \\ temperature experimental data relies on a sampling of the data that is \\ logarithmically spaced in time. The initial guesses for the thermal conductivity \\ values are obtained applying the well known and ideal model of the linear \\ temperature evolution versus the logarithm of the time. These values are used to \\ start up the algorithms that are applied in the minimization of the cost functional of \\ the squared residues between measured and calculated temperatures. The precision \\ of the estimates is assessed with the calculated confidence bounds obtained by the \\ variance-covariance matrix at the converged solution. \\ Vandoeuvre Lès Nancy Cedex, France \\ F. R. Pereira and N. Cella \\ Instituto Politécnico, IPRJ, \\ Universidade do Estado do Rio de Janeiro, UERJ, CP \\ 97282, 28601-970, Nova Friburgo, RJ, Brazil \\ cella@iprj.uerj.br

\section{A. J. Silva Neto} \\ Instituto Politécnico, IPRJ, \\ Universidade do Estado do Rio de Janeiro, UERJ, CP \\ 97282, 28601-970, Nova Friburgo, RJ, Brazil \\ Nuclear Engineering Program - COPPE, \\ Universidade Federal do Rio de Janeiro, UFRJ, CP \\ 68509, 21945-970, Rio de Janeiro,RJ, Brazil \\ ajsneto@lmn.con.ufrj.br
}

\section{INTRODUCTION}

The hot-wire technique (Norme Internationale, 1987, Davis, 1984) is generally used for the measurement of the low thermal conductivity of various materials, including powders, semi-transparent foams, and also for the characterization of glass. Since the early use of this method in thermal characterization, one can notice a persistent use of a direct model that relies on the simple and idealized hypothesis of a non participating role of both the hot wire and the thermocouples. Even though these conditions may be achieved for specific measurements, this is not always the case. The main purpose of this paper is to present a complete direct model that is able to take into account the resistive and capacitive effects of the wire, the possible existence of a contact resistance, the appearance of size limiting effects, when long times are considered so that the heat exchange with the surroundings of the sample has to be considered. The design of the present hot-wire apparatus is based on the objective of applying the technique at high temperatures, for porous ceramic materials and molten glasses, and is exposed in section 2. The resulting constraints impose the development of a direct model able to take into account all the non-ideal effects mentioned earlier. Section 3 presents thus the theoretical model, based on the quadrupole method. This method, generally poorly used, has been recently presented in the textbook of Maillet et al. (2000). It is well suited for the modeling of thermal experiments as it complies both with simple geometries, input-output matrix transfer formulation of the problem and fast computational times. It also extends the well known electrical analogy made in 


\section{CIÊNCIA/SCIENCE}

heat transfer to the transient regimes thus offering a very close physically related sense for the enginneer. In the fourth section, the strategy of the estimation procedure is presented, that underlines the fact that the use of a complete model makes the task of the inversion procedure more difficult, thus requiring the use of all the concepts developed in the theory of parameter estimation to assess the quality of the results. Section 5 then presents the inverse problem, discussing results obtained on the test case of a sample of paper (compressed journal paper) used as a test case for validating the overall procedure of thermal conductivity measurement.

\section{EXPERIMENTAL APPARATUS}

\subsection{Basis of the method}

The hot-wire technique consists in generating a heat transfer within a sample by the application of a sudden constant heat flux induced by Joule effect in a metallic wire imbedded in the sample. The geometry of the experiment considered is cylindrical with a onedimensional radial heat transfer (infinite length of the wire). The material that surrounds the wire is considered infinite in the radial direction, thus avoiding heat losses effects to be taken into account. In the version presented below, the temperature on the wire itself is measured and associated with the efficiency of heat transfer away from it, in the material. In the idealized conditions, this experiment leads to a linear evolution of the temperature of the wire as a function of the logarithm of the time, thus making the identification process very easy to implement and control.

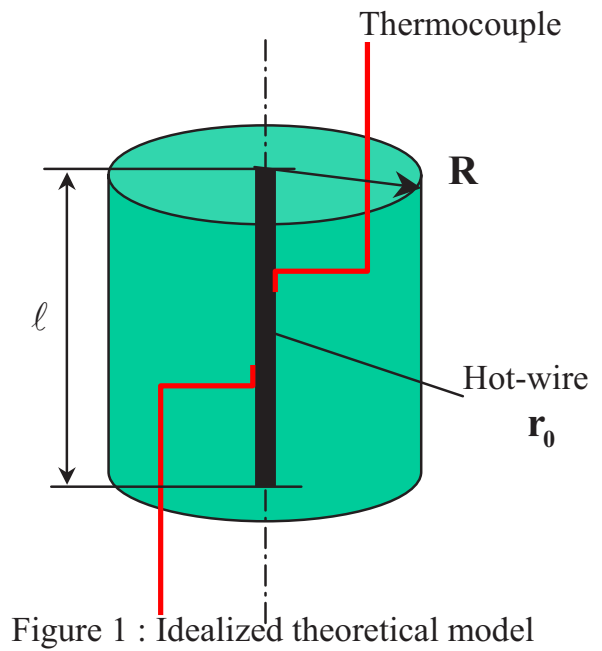

$$
\theta_{i}\left(r_{0}, t\right)=\frac{\phi}{4 \pi k \ell} \ln (t)+C^{t e}
$$

- $\mathrm{r}_{0}, \ell$ : radius and length of the wire

- $\mathrm{k}$ : thermal conductivity of the material

- $\theta_{i}, \phi$ : temperature and flux at radius $r_{0}$ of the wire

- $\quad C^{t e}$ : defined later in Eq. (10).

\section{S. André et al. Hot Wire Method For The Thermal...}

The system has been manufactured by Thermocoax ${ }^{\circledR}$. The hot wire is of $500 \mu \mathrm{m}$ of diameter, made of Inconel $(\mathrm{Ni} / \mathrm{Cr} 80 / 20)$. The properties of the wire are given in Table (1). The thermophysical properties are those given by the manufacturer and compare well with data from the literature. Two thermocouples have been laser-soldered, symmetrically from the mid-distance of the wire's length. These are $\mathrm{K}$ thermocouples (Chromel-Alumel), with Inconel shield of $1 \mathrm{~mm}$ of diameter, but thinned down over the last $1 \mathrm{~cm}$ of their extremity at $500 \mu \mathrm{m}$ where they are soldered on the wire (see Fig. (2a)). They allow to compare each of the measurements and to use the average measured value in the identification process. The hot wire is connected to pure Nickel wires in a specially designed connector made of ceramic (Stumatite) and involving a ceramic gliding bead and an excess dilatation wire. Then the electrical cables are connected to the constant stabilized current power supply, located beside the furnace. The hot wire resistance is of $0.662 \Omega(5.563 \Omega / \mathrm{m})$ and the resistance of the $3 \mathrm{~m}$ length part of the thick Nickel cables is $0.482 \Omega$. No significant heat power variations have been experimentally measured with this wire. The temperature of the wire given by the thermocouples is recorded through an $\mathrm{AD}$ converter (MCP3201, Microchip $\AA$, 8 channels, maximum sampling rate $100 \mathrm{kHz}, 12$ bits, $0.02^{\circ} \mathrm{C}$ resolution in the span $20-80^{\circ} \mathrm{C}$ ). A logarithmically spaced time acquisition has been programmed to generate small files (1000 points) starting at very low times (1 ms).

One hundred data points are acquired at equilibrium (reference state) before the controlling acquisition board triggers the power supply. The voltage and current (measured over a calibrated shunt resistance of Manganin) are recorded simultaneously allowing possible real time correction of the power variations on the signal. The electrical measured resistance of the hot-wire part in contact with the sample allows the calculation of the heat flux applied to the system.

\section{DIRECT MODELING}

\subsection{Necessity of a complete model}

The equipment described above has been designed to fulfill measurements in various conditions, especially high temperatures, and for various materials, including ceramic powders and molten glass which is highly corrosive. These constraints rule the design of the equipment which in its turn determines the validity of the hypothesis that can be made when using the direct model.

- The aim of characterizing powders implies a model that takes into account a thermal contact resistance between the wire and the sample into account;

- The high temperature conditions, furthermore in oxidizing conditions, lead to a choice of a laser welding of the thermocouples on the wire. The welding process has imposed minimum diameters for both the wire and the shielded thermocouples of 500 $\mu \mathrm{m}$, which is still thin compared to the dimension of the sample, but may alter the

\subsection{Technical specifications}


Table 1 : Properties of the hot wire

\begin{tabular}{|c|c|c|c|c|c|c|}
\hline$\ell$ & $\mathrm{r}_{0}$ & $\mathrm{kw}$ & $(\rho \mathrm{C})_{w}$ & $\alpha_{w}$ & $\mathrm{C}_{w}=(\rho \mathrm{CV})_{w}$ & $\mathrm{R}_{w}=1 / 8 \pi \mathrm{k}_{w} \ell$ \\
\hline 120 & 250 & 13.4 & 3.868 .600 & $3.46410-6$ & 0.0912 & 0.0247 \\
$(\mathrm{~mm})$ & $(\square \mathrm{m})$ & $(\mathrm{W} . \mathrm{m}-1 . \mathrm{K}-1)$ & $(\mathrm{J} . \mathrm{m}-3 . \mathrm{K}-1)$ & $\left(\mathrm{m}^{2} . \mathrm{s}-1\right)$ & $(\mathrm{J} . \mathrm{K}-1)$ & $(\mathrm{K} . \mathrm{W}-1)$ \\
\hline
\end{tabular}

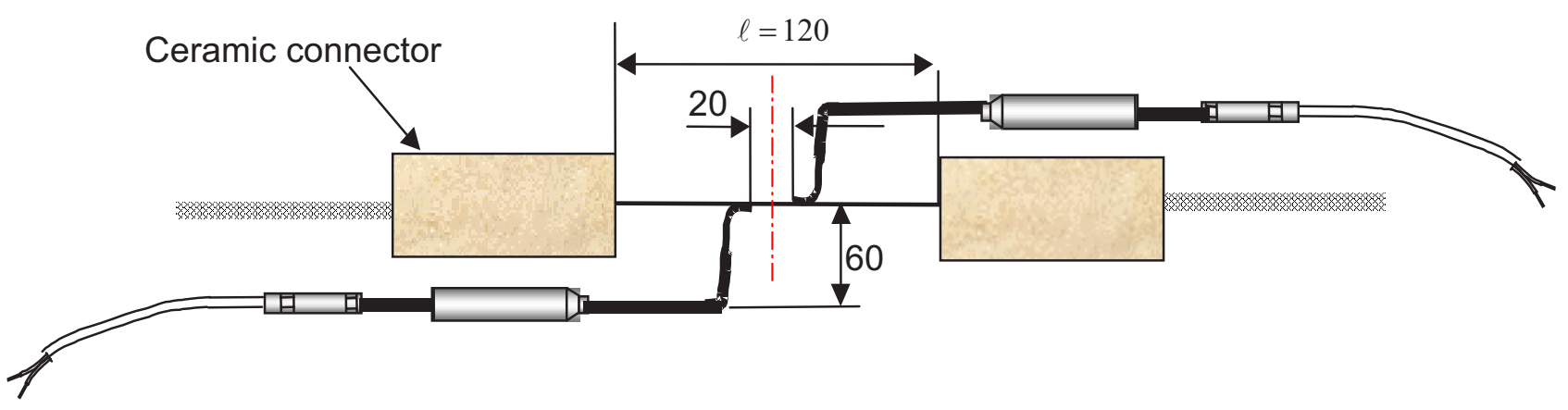

Figure 2a : Hot-wire, connections with power supply cables and thermocouples (all dimensions in $\mathrm{mm}$ ).

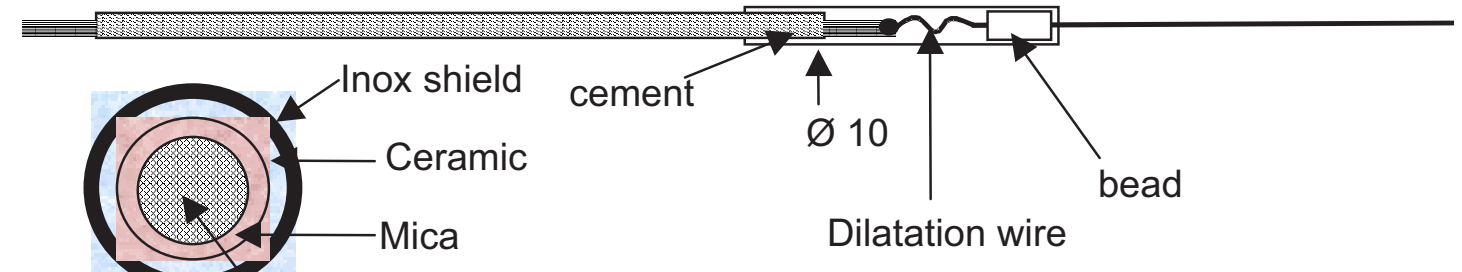

Multifilar Nickel wire

Figure $2 \mathrm{~b}$ : Ceramic connector, transversal view of the connecting cables.

thermograms at short times mainly because of bulk capacitive effects;

- The application to ceramic materials processed through syntherisation does not allow the manufacturing of samples of large diameter (maximum of $3 \mathrm{~cm}$ of radius) thus making the hypothesis of the semi-infinite medium not valid. So it is when glass is considered where the additional mechanism of radiative transfer reaches the boundaries of the sample very early (In this latter case, it is obviously necessary to have a direct model with coupled conduction and radiation modeling of heat transfer, which is not the case in this paper).

All these aspects have been embedded in the construction of the direct model. The quadrupole method described in next section takes them into account without any complications and makes this method powerful. Furthermore the solution so obtained is analytical. It is important to note already that having such a model at hand does not mean that all of these features will be simultaneously turned on for any identification procedure. It simply means that a complete versatility can be achieved when working with the hot-wire technique. It is in turn the scientist that performs the measurement that has to determine which effects may be encountered in a given experiment. According to this analysis, various reduced models can be obtained from the complete one, that sets the dimension of the parameter vector to be estimated. It is with this principle in mind that the identification software has been developed and that the example shown in section 5 has been chosen.

\subsection{Quadrupole method}

The method has received a full description in a recent textbook (Maillet et al., 2000) that the interested reader may consult for a more detailed derivation of the calculations presented here.

The method originates in the integral transform treatment of the heat equation. As far as one dimensional heat transfer is considered in only one direction but in transient regime, the integral transform under consideration is the Laplace transform in time. Starting from zero initial conditions (equilibrium reference state), the partial derivative heat equation is transformed into a purely linear differential equation. Then one recovers an input-output matrix transfer formulation of the problem, well known from scientists working in the electronic field (automation systems). The variables embedded in the formulation are the temperature (potential) and the heat flux (current) 


\section{CIÊNCIA/SCIENCE}

according to the well known electrical analogy. In terms of vector formulation, this leads for one layer of material to

$$
\left(\begin{array}{l}
\theta \\
\phi
\end{array}\right)_{\text {in }}=\left(\begin{array}{ll}
A & B \\
C & D
\end{array}\right)\left(\begin{array}{l}
\theta \\
\phi
\end{array}\right)_{\text {out }}
$$

where $A, B, C, D$ are the coefficients of the quadrupole, that depends on $p$, (the Laplace variable), and whose formal expressions depend on the geometry at hand. $\theta$ and $\phi$ represent now the Laplace transforms of the temperature and heat flux variables. It is worthwhile to note here the intrinsic character of this formulation which until now never assumes to know the thermal boundary conditions of the problem. Classically, two of them have to be precise to derive the two other unknowns. Anticipating the results derived later on, if one considers that relation (2) represents the hot-wire experiment, one will introduce $\phi_{i n}=\frac{\phi_{w}}{p}$, Laplace transform of the Heaviside function with dissipated power $\phi_{w}, \phi_{\text {out }}=0$, if a finite layer is considered with adiabatic conditions on the external surface, and the exact direct model of the thermogram measured on the wire is simply, in Laplace domain,

$$
\theta_{\text {in }}=\frac{A(p)}{C(p)} \frac{\phi_{w}}{p}
$$

Since the 70's, very fast and precise algorithms have been developed allowing the numerical return to the time domain (see Maillet et all, (2000) for an introductory review).

The electrical analogy is then extended to transient heat transfer problems. The consequence of Eq.(2) is that an electrical scheme involving three impedances connected in a $\mathrm{T}$ or $\Pi$ network can be classically derived according to the sketch shown in Fig. (3) where a T-network is considered.

The expression of the impedances can be related to the four coefficients of the matrix transfer as shown in Eq. (4)

$$
Z_{1}=\frac{A-1}{C}, Z_{2}=\frac{D-1}{C}, \quad \text { and } Z_{3}=\frac{1}{C}
$$

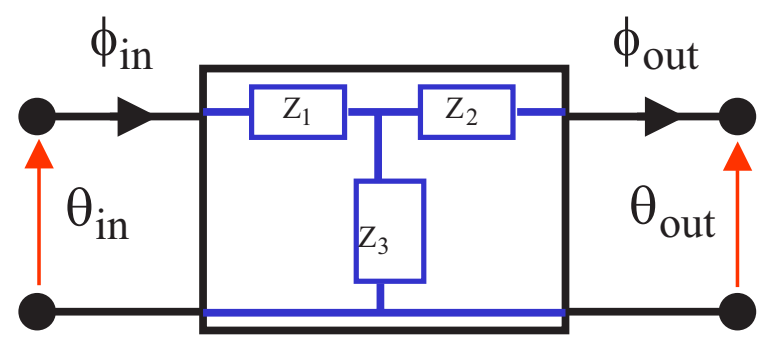

Figure 3 : Quadrupole or "Three impedances" network associated to a layer of material.

When a material layer can be reduced to a pure resistive behavior, as it is in steady-state regime, one has the matrix transfer $A=1, B=R_{t h}$ (thermal resistance of the layer), $C=0$ and $D=1$, which is nothing else than Ohm's

\section{S. André et al. Hot Wire Method For The Thermal...}

law. One would also demonstrate in a very general way, that asymptotic development of the formal expressions of $Z_{1}, Z_{2}$, and $Z_{3}$ for small $p$ values (infinite times, i.e. steady state equilibrium) makes expressions of $Z_{1}$ and $Z_{2}$ to be pure resistance (depending only on $k$ ) and $Z_{3}$ to be a pure capacity (depending only on the heat capacity of the medium).

Thus the quadrupole method bridges the gap between the resistance of the steady-state problem and the heat capacity of the lumped body approximation.

\subsection{Hot-wire experiment modeled with quadrupoles}

We give now without demonstrations the mathematical relations and electrical scheme corresponding to the hotwire experiment modeling.

- For the cylindrical layer, the coefficients of the quadrupole are

$$
\begin{aligned}
& A=s R\left(K_{1}(s R) I_{0}\left(s r_{0}\right)+K_{0}\left(s r_{0}\right) I_{1}(s R)\right) \\
& B=\frac{1}{2 \pi k \ell}\left(K_{0}(s R) I_{0}\left(s r_{0}\right)-K_{0}\left(s r_{0}\right) I_{0}(s R)\right) \\
& C=-2 \pi k \ell s^{2} R r_{0}\left(K_{1}(s R) I_{1}\left(s r_{0}\right)-K_{1}\left(s r_{0}\right) I_{1}(s R)\right) \\
& D=s r_{0}\left(K_{0}(s R) I_{1}\left(s r_{0}\right)+K_{1}\left(s r_{0}\right) I_{0}(s R)\right)
\end{aligned}
$$

where $K_{n}(r)$ and $I_{n}(r)$ are the modified Bessel functions, and $s$ corresponds to $\sqrt{p / \alpha}$, where $\alpha$ is the diffusivity of the material.

Equations (5) are suited for the cylindrical layer of the material to characterized.

- Similar expressions can be obtained for a cylindrical heating element (the Hot-wire) defined by its averaged Laplace temperature

$\theta_{m}=\frac{1}{\pi r_{0}^{2} \ell} \int_{0}^{r_{0}} \theta 2 \pi r \ell d r=\frac{2}{r_{0}^{2}} \int_{0}^{r_{0}} \theta r d r \quad$ and mean power dissipation $\phi_{m}=\pi r_{0}^{2} \ell L(g(t))$, where $L(g(t))$ is the Laplace transform of the volumetric heat dissipation (generally noted ' $g$ ' in the heat equation). But a first simplification can be introduced here due to the generally small size of the wire compared to the material, that allows to consider responses time weak compared to the medium ones. In the asymptotic development $\left(\mathrm{p} \rightarrow 0\right.$ and $\left.\mathrm{r}_{0}<<\mathrm{R}\right)$, one would obtain for the wire :

$Z_{1}=0$

$Z_{2}=\frac{1}{8 \pi k_{\mathrm{w}} \ell}=R_{\mathrm{w}}$

$Z_{3}=\frac{1}{\pi r_{0}^{2} \ell\left(\rho C_{p}\right)_{w} p}=\frac{1}{C_{\mathrm{w}} p}$ with $C_{\mathrm{w}}=\pi r_{0}^{2} \ell\left(\rho C_{p}\right)_{\mathrm{w}}$

This means that the behavior of the wire, within the approximation mentioned above, can be reduced to pure resistive $\left(\mathrm{R}_{w}\right)$ and capacitive $\left(\mathrm{C}_{w}\right)$ effects. 


\section{CIÊNCIA/SCIENCE}

- The heat losses are represented by a pure convective resistance $\mathrm{R}_{h l}$ :

$R_{h l}=\frac{1}{h S} \quad$ with $S=2 \pi R \ell$

where $h$ is the heat transfer coefficient.

- Finally, we have the quadrupole corresponding to a cylindrical 'layer' which may be regarded as infinite $(\mathrm{R} \rightarrow \infty)$. One obtains then $\mathrm{Z}_{2}=\mathrm{Z}_{3}=0$ and $\mathrm{Z}_{1}$ reduces to the simple relation :

$Z_{1}=Z_{\infty}=\frac{1}{2 \pi k \ell} \frac{K_{0}\left(\mathrm{sr}_{0}\right)}{\mathrm{sr}_{0} K_{1}\left(\mathrm{sr}_{0}\right)}$

and the electrical scheme that corresponds to this situation is readily obtained (Fig. (4)).

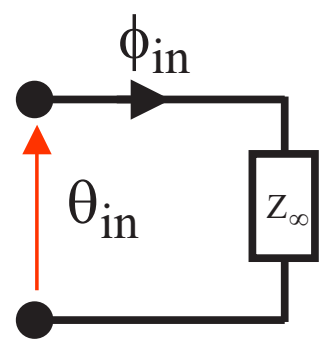

Figure 4 : Quadrupole representation of the semi-infinite layer.

- The complete model.

The hot-wire experiment is fully described according to the sketch of Fig. (5), made of all the elementary bricks defined in the above Eqs. (5-8). The reduction of the model from the finite case to the semi-infinite medium is obtained from Fig. (5) by simply substituting the last two 'bricks' (Finite medium and heat losses) by the semiinfinite impedance of Fig. (4). The model can be analytically expressed as
S. André et al. Hot Wire Method For The Thermal...

$\theta_{1}(\mathrm{p})=\frac{\phi_{m} / p}{\left(\frac{1+R_{\mathrm{w}} C_{\mathrm{w}} p}{R_{\mathrm{c}}+Z^{\prime}}\right)}$ with $Z^{\prime}=Z_{\infty}($ semi - infinite case $)$

or $\quad Z^{\prime}=Z_{1}+\frac{Z_{3}\left(Z_{2}+R_{\mathrm{hl}}\right)}{\left(Z_{3}+Z_{2}+R_{\mathrm{hl}}\right)}$

Finally, thanks to this complete model, one can develop the exact solution for long times $(p \rightarrow 0)$. This asymptotic development in the case of the semi-infinite medium leads to the determination of the constant of Eq. (1). One finds

$C^{\text {te }}=\phi_{m} R_{T}-\frac{\phi_{m}}{4 \pi k \ell} \gamma_{e}-\frac{\phi_{m}}{4 \pi k \ell} \ln \left(\frac{r_{0}^{2}}{4 \alpha}\right)$

with $\gamma_{\mathrm{e}}=0.57721$ is the Euler constant, and $R_{T}=R_{\mathrm{c}}+R_{\mathrm{w}}$.

A linear regression on the correctly identified linear part of the thermogram then provides initial guesses for two parameters. The thermal conductivity of the medium $\mathrm{k}$ is obtained from the slope. If for example, the contact resistance is expected to be negligible, $R_{T}$ reduces to the resistance of the wire $\mathrm{R}_{w}$ (Table (1)) and the parameter $\mathrm{t}_{\mathrm{c}}=\mathrm{r}_{0}^{2} / \alpha$ can be estimated from Eq. (10) and used as starting guess in the numerical procedure.

\subsection{Direct simulations - Knowledge of the physics of the problem}

We present in Figs. (6) and (7) the effects of contact resistance, hot-wire heat capacity and heat losses. Dimensionless quantities are used. The thermograms shown correspond to the dimensionless temperature $\theta_{1}^{*}=\frac{\theta_{1}}{\left(\phi_{m} / \pi k \ell\right)}$ versus the logarithm of the dimensionless time $t^{*}=\alpha t / R^{2}$. Therefore, the thermograms are plotted in log-space for the abscissa.

In Fig. (6), we plot the influence of the dimensionless contact resistance $R^{*}=\pi k \ell R_{\mathrm{c}}$ on the thermograms. The analysis of Fig. (6) shows that the

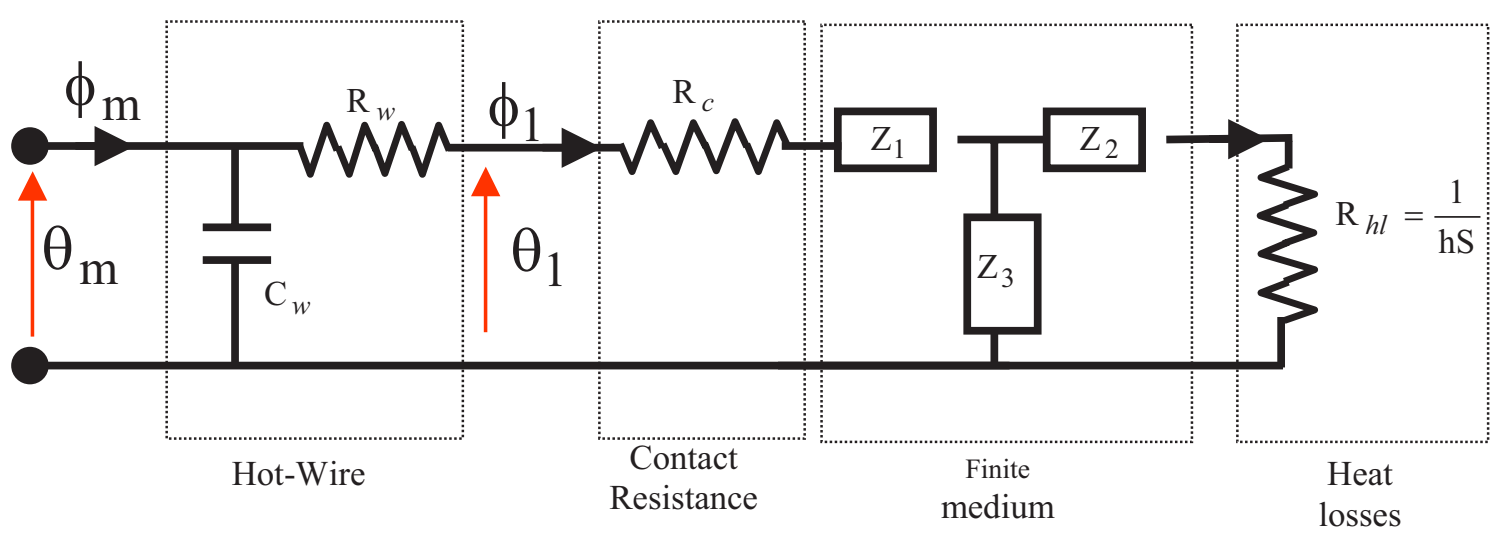

Figure 5 : Analog representation of the hot-wire experiment. 


\section{CIÊNCIA/SCIENCE}

absence of contact resistance leads to a perfect linear evolution of the temperature. The greater the contact resistance is, the shorter the linear part of the thermogram becomes along with an increase in the temperature reached by the wire (insulation effect).

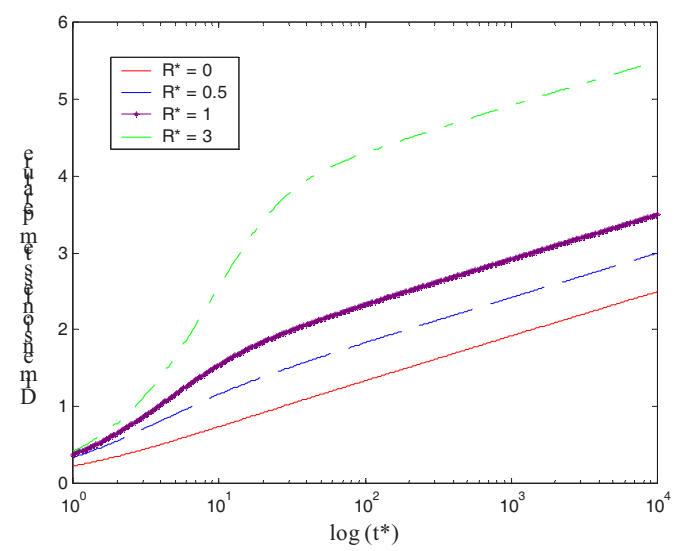

Figure 6 : Effect of the total reduced resistance

$$
\left(C^{*}=2.15\right) \text {. }
$$

In Fig. 7, we show the influence of both the capacity of the wire and the heat losses effect (finite medium). Thermograms are given for various values of the dimensionless capacity $\mathrm{C}^{*}=(\rho \mathrm{C})_{w} /(\rho \mathrm{C})_{\text {medium }}$. For the curve obtained for $\mathrm{C}^{*}=5$, we show the effect of the finite medium for different values of the Biot number $\mathrm{H}=\mathrm{hR} / \mathrm{k}$, respectively $\mathrm{H}=0, \mathrm{H}=10$ and $\mathrm{H}=\infty$. The external radius considered here is $\mathrm{R}=3 \mathrm{~cm}$ which corresponds to a reduced ratio of $e^{*}=\left(R-r_{0}\right) / \mathrm{r}_{0} \approx 100$.

What can be observed from these curves is firstly that the heat capacity of the wire should be as small as possible because it strongly affects the early time evolution of the temperature rise, just as the thermal resistance does. It has nevertheless no influence on the later temperature rise. As could be expected from a capacity element in an electrical network, it acts as a delay for the dynamical system. Secondly, one can note that the influence of the heat losses is only sensible in the long times part of the thermogram.

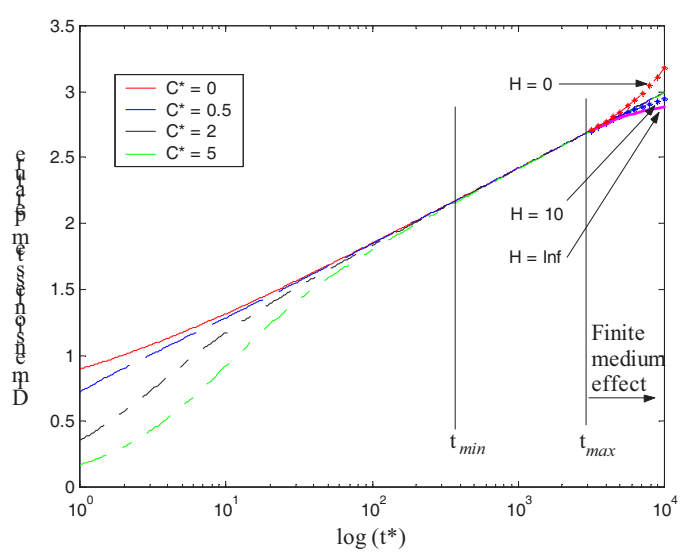

Figure 7 : Effect of the hot-wire heat capacity and of the Biot number $\left(\mathrm{R}^{*}=0.5\right)$.

\section{S. André et al. Hot Wire Method For The Thermal...}

The main conclusion that can be drawn from the simulations presented in Figs (6) and (7), is that all the non-idealized effects (hot-wire capacity effect, thermal contact resistance, and finite medium effect) reduced the linear part of the thermogram. As shown earlier in section 3.1, all these effects may arise in an experiment, and the complete model presented above can be used either directly in an estimation procedure, or indirectly, by defining two times $\mathrm{t}_{\min }$ and $\mathrm{t}_{\max }$ bounding the time interval on which the linear behavior model given by Eq. (1) can be used. One can also state that insufficient concern in the physics involved may lead the experimentalist to stop the experiment too early, thus working on the part of the thermogram that reflects the effect of heat capacity of the wire, and possibly contact resistance, instead of the medium's thermal conductivity. A plot on a logarithmic scale in this restricted domain of time exhibits also a linear evolution that may be confused with the 'good' one, thus leading to substantially underestimate what is supposed to be the thermal conductivity parameter.

\section{The inverse problem}

According to the model presented in section 3.3, the parameter vector exhibits a maximum of 5 parameters

$\beta=\left\{\beta_{1}=\mathrm{k}, \beta_{2}=\mathrm{C}_{w}, \beta_{3}=\mathrm{t}_{\mathrm{c}}=\mathrm{r}_{0}^{2} / \alpha, \beta_{4}=\mathrm{R}_{\mathrm{T}}, \beta_{5}=\mathrm{h}\right\}$

Such an inverse problem has already been investigated by Zhang (1993), Zhang et al. (1993) and Rémy and Degiovanni (2001). From these previous studies relating a sensitivity analysis of the Parameter Estimation Problem (PEP), one can retain firstly that the thermal conductivity of the medium, the parameter that is aimed to identify, is the parameter of highest sensitivity and generally sufficiently uncorrelated from the other parameters. Secondly, the sensitivity analysis justifies the model reduction embedded in the direct model presented above, that considered only the capacitive effect of the wire. The diffusivity and conductivity of the wire can be proved to be totally correlated. Only the heat capacity has to be taken into account, the resistance of a metallic wire $\mathrm{R}_{w}$, always small, can be considered as a known parameter and in all cases can not be discriminated from the contact resistance, if it exists. Despite these two remarks, the PEP for the hot wire conductivity is still complicate due to modifications of the correlation coefficients between the parameters of the model, that depends of the nominal values. Calculations of sensitivity coefficients or expected variance-covariance matrix of the parameters show that in some cases $\beta_{4}$ (contact resistance parameter) may appear totally correlated with $\beta_{3}=t_{c}$ (diffusivity of the medium). In other cases, i.e. when no contact resistance is considered, it is $\beta_{2}$ (hot-wire capacity parameter) that may in some cases be correlated with $\beta_{3}$.

Our purpose in this section is to give an example which aims to be a pedagogical one, showing that if a 'blind-type' estimation is carried on using the most sophisticated model and performing minimization 


\section{CIÊNCIA/SCIENCE}

algorithms, large errors can be made. On one hand, one has a complete model (synonymous with large parameter vector) able to take into account different physical phenomena. On the other hand, one has a given experiment, on a given sample with specific constraints. One has to perform the identification making these two things in perfect symbiosis.

\subsection{Fitting procedure}

In this section, instead of recalling all the tools involved classically in PEP and developed in many good textbooks like for example the one of Beck and Arnold (1977), we simply give the list of all the features available in the software package developed for post-data treatment under MATLAB environment.

- $\quad$ setting the data for the estimation procedure (statistical analysis of the temperature recordings at equilibrium i.e. characterization of the noise, calculations of the heat power dissipated in the wire and control of stability, possible reduction of the number of data points, plot of the thermograms, dissipated power, temperature of cold junction...)

- Initialization of all needed data.

- Selection of the model type (semi-infinite or finite medium).

- Selection of the model (and parameter vector). Five different models are available ranking from a 1 parameter model to 4 parameter model (dimensionless or not).

- Clicking mouse selection of the linear part of the thermogram on which the user wishes to calculate initial values for 2 parameters according to Eqs (1) and (10).

- Possibility of taking the thermocouple into account by adding a parameter which is the characteristic response time of the thermocouple ( $\mathrm{R}-\mathrm{C}$ branch).

- Selection of the minimization algorithm:

Estimations are performed in the classical way of minimizing the uni-modal cost functional that consists on the square of the temperature residuals, i.e. distance between experimental and calculated thermograms (OLS estimator). Three inversion algorithms have been considered in the estimation software :

- the simplex Nelder-Mead and LevenbergMarquardt algorithms, based on a deterministic search of the minimum. These methods are very efficient in terms of computational time. In some complicated cases where a good design of the PEP can not be achieved (i.e. in case of correlated parameters), such methods lead to local minima that are dependent upon the initial guesses. This induces an error on all the estimates, according to the influence of the noise on the sensitivity matrix. We use the built-in function fminsearch for the Nelder-Mead simplex algorithm, of the function leasqr.m developed by Richard I. Shrager, from the National Institute of Health (Bethesda, MD, USA) and modified by Arthur Jutan and Ray Muzic, available at the Internet Matlab site (matlab42/toolbox/contrib).

- the stochastic optimization (exhaustive search) algorithm used is the "Simulated Annealing"
S. André et al. Hot Wire Method For The Thermal...

method (SA), which has been already used in heat transfer problems by Silva Neto and Soeiro (2002, 2003). This method is less sensitive than the previous deterministic algorithms in the case of problems being somewhat badly conditioned. On a computational point of view, this method requires prohibitive time for data treatment. It has been compulsorily used in this study as it provides an alternate method for securing the results, but mainly because it allows to constrain the parameter search as it operates on a pre-defined domain of the parameter space. We used function SimAnneal.m developed by Peter Cervelli from the Dept. of Geophysics (Stanford University).

- Results : The software provides the calculations of the sensitivity coefficients and sensitivity matrix at convergence and for the initial parameter vector. It calculates also the optimal cost expected from the characteristic of the noise of the signal, the theoretical variance-covariance matrix expected from the characteristic of the noise, the effective variancecovariance matrix calculated at convergence taking the residuals into account instead of the noise, and the $95 \%$ confidence interval.

\subsection{Example of estimation}

In Fig. (8) we show an experimental thermogram obtained on a sample of compressed journal paper. Curves denoted 1 correspond to the hot wire thermogram (thermocouples 1 and 2 recordings, with their average value) that has been offset with respect to the base line signal recorded for negative times (equilibrium reference state). Curve 2 corresponds to the Cold-Junction temperature that undergoes an undesired heating and may alter the temperature recordings as can be seen from the temperature rise on curve 3 (temperature measured on the external surface of the sample). It can not correspond to size limited effect in view of the times involved. This will be a source of bias in the residuals as shown later. Curve 4 (given here without units specification) corresponds to the recording of the power dissipated in the wire and is shown to be perfectly stable even at the very short times (not shown here for clarity of the figure).

Estimations are now performed with the complete model in the semi-infinite case. Care has been taken in order to reduce possible contact resistance to zero by performing a good immersion of the wire and thermocouples at the plane surface of the two-parts sample. The initial value considered for parameter $\beta_{4}=\mathrm{R}_{\mathrm{T}} \cong \mathrm{R}_{w}$ is given in Table 1 . So it is for the capacity of the wire from the manufacturer values. The thermal conductivity and characteristic time are estimated using Eqs. (1) and (10) using a regression fitting in the linear part of the thermogram ranging approximately from $t_{\text {min }}=200 \mathrm{~s}$ to $t_{\max }=1100 \mathrm{~s}$ (manual selection). One obtains generally an initial conductivity value $\mathrm{k}_{\mathrm{i}}=0.1$ and an initial value of $t_{c_{i}}=1.25$ with some $\pm 20 \%$ of departure around this value. The medium has a measured density of $\rho=215 \mathrm{~kg} \cdot \mathrm{m}^{-3}$, and an unknown specific heat. 


\section{CIÊNCIA/SCIENCE}

Assuming a value of the order of $C_{p}=1400 \mathrm{~J} . \mathrm{kg}^{-1} \cdot \mathrm{K}^{-1}$

(classical value for paper in the literature), we may therefore expect a diffusivity value of the order of $\alpha=0.33 \mathrm{~mm}^{2} . \mathrm{s}^{-1}$ and a characteristic time of the order of $t_{c}=0.2 \mathrm{~s}$.

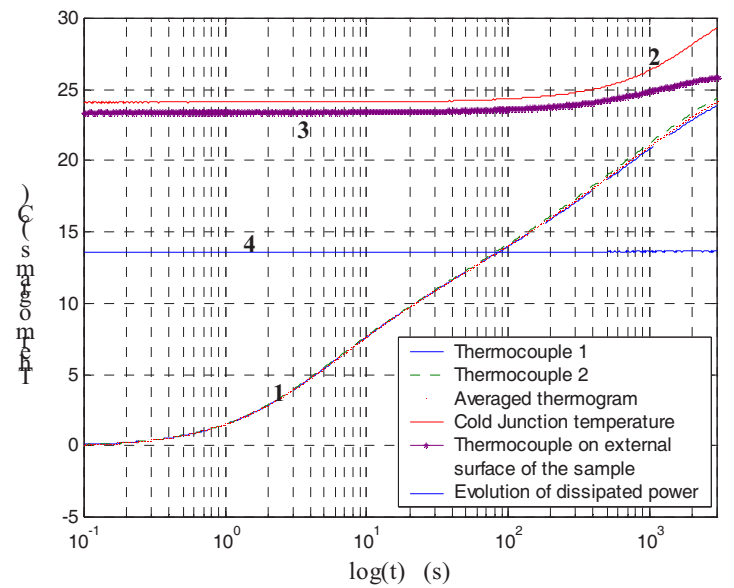

Figure 8 : Experimental thermograms.

Applying the Levenberg-Marquardt algorithm yields the results shown in Fig. (9). The values obtained for the 4 parameters in the order specified by Eq. (11) are given in the figure caption. The identification is perfect exhibiting residuals less than $0.1{ }^{\circ} \mathrm{C}$ over the entire time interval and a conductivity value of 0.1 , the value found with the ideal model of Eq. (1). One may feel satisfied with this result except that if one looks at the values obtained for the other parameters, the identification seems to have failed. It is impossible considering our knowledge of the experiment to get a resistance value of $R_{T}=11.8 \mathrm{~K} / \mathrm{W}$ and a characteristic time of $t_{c}=9 \mathrm{~s}$, which leads to a very small diffusivity and then to an unacceptable value of $C_{P}$.

In fact, the LM algorithm has been trapped into a local minimum simply because the model used was overparameterized. Maintaining parameter $\mathrm{R}_{\mathrm{T}}$ (known to be negligible and thus having a null sensitivity) in the parameter vector, gives all orthogonality to the algorithm to deal with the data and find a perfect optimum in terms of the cost function to minimize.

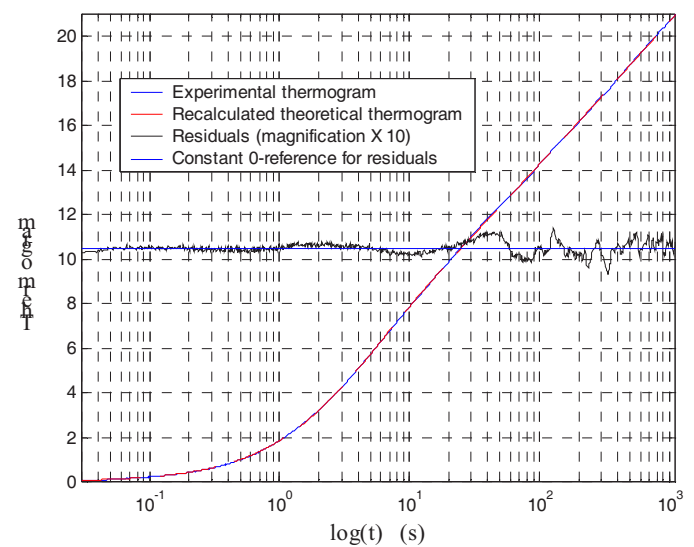

\section{S. André et al. Hot Wire Method For The Thermal...}

Figure 9 : Identification performed with complete model and Levenberg-Marquardt algorithm. (Number of iterations $=22$, $\beta_{1}=0.100, \beta_{2}=0.196, \beta_{3}=t_{c}=9.46$, $\beta_{4}=11.78, \quad$ Cost $=0.74$ )

On the other hand, using the Nelder-Mead simplex algorithm leads to the following results (Fig. (10)) :

The conductivity value is now of $k=0.11$ and all the other parameters have reasonable values compared to our knowledge of what should be. The residuals are not as good as those obtained using the LM algorithm (of the order of $0.4^{\circ} \mathrm{C}$ ) but this is due to the fact that a bias clearly exists between model and experiment (large oscillations). Such a bias may find acceptable explanations that for some of them can lead to slight modification of the experiment. For example in our case, one has to verify that the cold junction temperature evolution during the experiment does not affect the temperature measurement (good correction). The parameter $\beta_{2}=C_{\mathrm{w}}$ is always overestimated which can be understood because at the measurement point, there is an excess of matter due to the thermocouple itself (of diameter $500 \mu \mathrm{m}$ soldered on a $1 \mathrm{~cm}$ length). The parameter $\beta_{3}$ is between the value found applying the asymptotic model of Eq.(1) and the value 'expected' from the literature value of $C_{p}$. Finally, one can note that the parameter $\beta_{4}, R_{T}$, is found to have an expected small value. It is a trend already observed in minimization of illposed problems that the Nelder-Mead simplex algorithm is less sensitive to a parameter having a very low sensitivity as is the case in our example. Furthermore, if the parameter vector was not initialized according to the procedure explained above (i.e. with somewhat arbitrarily choice), the algorithm also fails, just as Levenberg-Marquardt does. This in turn means that the direct model can not be used with the full parameter vector. The last comment is that the conductivity value is different within $10 \%$ depending on the minimization algorithm used and this is not acceptable.

Now we use the model by setting parameter $R_{T}$ to the fixed value $R_{w}$ and performing the identification with a parameter vector of 3 components : $\beta=\left\{\beta_{1}=k, \beta_{2}=C_{\mathrm{w}}, \beta_{3}=t_{c}=r_{0}^{2} / \alpha\right\}$. In this case, the result obtained is exactly the same as the one shown in Fig. (10) and does not dependent on the algorithm used (results therefore not presented here). The fact that the two algorithms converge now to the same estimates, being more insensitive to the initial conditions, is considered to be a proof of the proper suiting of both the direct model and the identification procedure. 


\section{CIÊNCIA/SCIENCE}

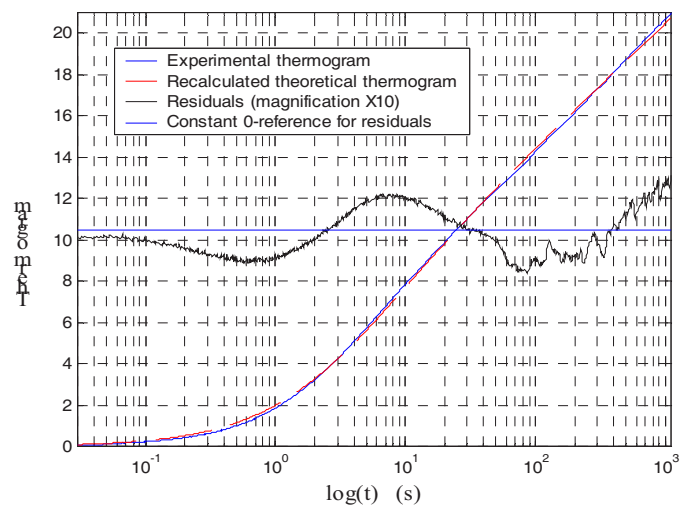

Figure 10 : Identification performed with complete model and Nelder-Mead simplex algorithm.

(Number of iterations $=220$,

$\beta_{1}=0.111, \beta_{2}=0.138, \beta_{3}=t_{c}=0.755$,

$\beta_{4}=0.019, \quad$ Cost $=13.2$ )

Figure 11 shows the behavior of the reduced sensitivity coefficients $X_{i}=\beta_{i} \frac{\partial T(t, \beta)}{\partial \beta_{i}}$ calculated using the estimates obtained for the unknowns.

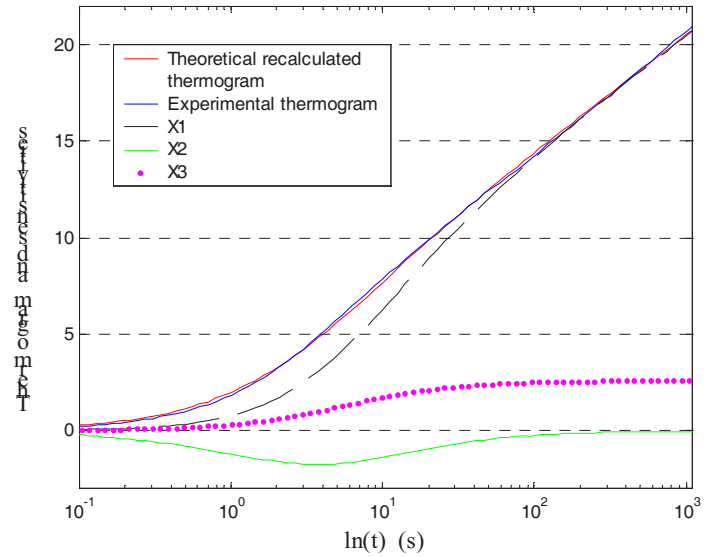

Figure 11 : Thermograms and reduced sensitivity coefficients for the case

$$
\beta_{1}=0.11, \beta_{2}=0.14, \beta_{3}=t_{c}=0.76 \text {. }
$$

The variance-covariance matrix calculated at convergence leads to the following variances on the estimates: $V\left(\beta_{1}\right)=0.64 \%, V\left(\beta_{2}\right)=5.3 \%, V\left(\beta_{3}\right)=25 \%$, showing that as expected, it is the thermal conductivity that has the smallest confidence interval. The correlation coefficients found are $\rho_{12}=0.71, \quad \rho_{13}=0.985, \quad \rho_{23}=0.76$ which is compatible with an accurate solution according to the Gallant criterion (Beck and Arnold, 1977):

Number of significant figures in the calculation $\geq 2 \log \left(\frac{\lambda_{\max }}{\lambda_{\min }}\right)=11$ with $\lambda_{\text {max }}, \lambda_{\text {min }}$ being the largest and smallest eigenvalues of the correlation matrix.

We plot also in Fig. (12) each sensitivity coefficient as a function of another in order to verify if any linear dependence exists between the 3 sensitivities. One must note that, compared to the two other combinations involving $\beta_{1}$, the two parameters $X_{2}$ and $X_{3}$ shows a more constrained dependence (due to smaller sensitivity values as for $\beta_{1}$ ) that tends to a quasi linear dependence only in a small region of time centered on the extremum of sensitivity of $\beta_{2}$ that corresponds also to the inflexion point of the sensitivity evolution of $\beta_{3}$. Therefore, even if the correlation coefficient $\rho_{13}$ between both does not indicate a high correlation, this global information is somewhat counterbalanced when analyzing carefully the sensitivity coefficients. This explains the highest variances obtained for these two parameters.

In all cases, it is absolutely impossible to identify correctly other properties than the thermal conductivity of the medium as far as the hot wire experiment is concerned with such a material.

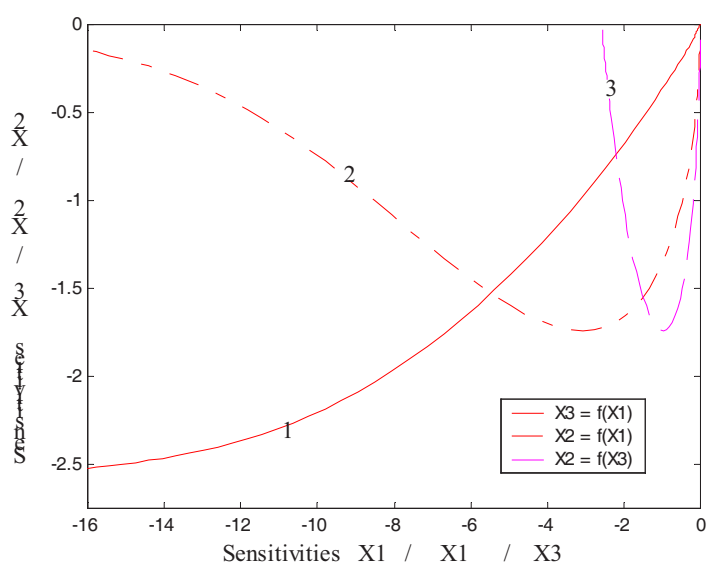

Figure 12 : Reduced sensitivity coefficients for the case $\beta_{1}=0.11, \beta_{2}=0.14, \beta_{3}=t_{c}=0.76$.

\section{Conclusion}

In this paper, we aimed to demonstrate that the modeling of an experiment needs to be as complete and precise as possible. But this does not mean that it has to be used without a great knowledge of the characteristic of the experiment really developed. Therefore, special care must be paid when performing the estimation and reducing the model (and the parameter vector as a consequence) accordingly to the physics really embedded. For this purpose the well known tools developed in the parameter estimation theory need to be used and introduced in the estimation software to allow objective means of analyzing the results. If this basic principle is applied, identification can be performed independently of the minimization algorithms used. If not, large errors may be obtained. This principle is simple and has to be clear for engineers or scientists involved in the metrology of thermal properties.

\section{Acknowledgements}

The authors acknowledge the financial support provided by FAPERJ and CNPq through research grants, and the company Unisafe from Nova Friburgo, Brazil, for the compressed journal paper samples. The first author acknowledges also $\mathrm{CNPq}$ for the Visiting Professor Scholarship. 


\section{References}

Beck, J.V., Arnold, K.J., 1977, "Parameter Estimation in Engineering and Science", NY, John Wiley \& Sons.

Davies, W.R., 1984, "Hot Wire Method for the Measurement of the Thermal Conductivity of Refractory Materials". N.Y., Compendium of Thermophysical Property Measurement Methods, Vol. 1, Edited by K.D. Maglic, A. Cezailigan and V.E. Peletsky, Plenum Press, pp. 231-254.

Maillet, D., André, S., Batsale, J.C., Degiovanni, A. and Moyne, C.,2000, "Thermal Quadrupoles : Solving the Heat Equation through Integral Transforms. Chichester, PA, John Wiley \& Sons Ltd.

Norme Internationale, 1987, "Matériaux Réfractaires - Détermination de la Conductivité Thermique - Partie 1 / Méthode du Fil Chaud I.S.O. 8894-1".

Rémy, B. and Degiovanni, A., 2001, "Inverse Method Applied to the Measurement of the Thermal Conductivity of Powders by a Hot Wire Technique", Proc. $9^{\text {th }}$ Int. Thermal Conductivity conference, Vol.19, No. 3, pp. 332-340.

Silva Neto, A. J. and Soeiro, F. J. C. P., 2002, "Estimation of Phase Function of Anisotropic Scattering with a Combination of Gradient Based and Stochastic Global Optimization Methods", $5^{\text {th }}$ World Congress on Computational Mechanics, Vienna, Austria.

Silva Neto, A. J. and Soeiro, F. J.C.P, 2003, "Solution of Implicitly Formulated Inverse Heat Transfer Problems with Hybrid Methods", Mini-Symposium Inverse Problems from Thermal/Fluids and Solid Mechanics Applications $2^{\text {nd }}$ MIT Conference on Computational Fluid and Solid Mechanics, Cambridge, EUA.

Zhang, X., Degiovanni, A. and Maillet, D., 1993, "Hot Wire Measurement of Thermal Conductivity of Solids : a New Approach", High Temp. - High Pressures, Vol.25, pp. 577-584.

Zhang, X., 1993, "Métrologie Thermique par Méthode Quasi-Stationnaire : Modélisation, Identification et Application à la Caractérisation de Solides", Thèse de Doctorat, INP Lorraine, Nancy, France. 